\title{
Brachial-ankle pulse wave velocity predicts all-cause mortality in the general population: findings from the Takashima study, Japan
}

\author{
Tanvir Chowdhury Turin ${ }^{1}$, Yoshikuni Kita ${ }^{1}$, Nahid Rumana ${ }^{1}$, Naoyuki Takashima ${ }^{1,2}$, Aya Kadota ${ }^{1}$, \\ Kenji Matsui $^{3}$, Hideki Sugihara ${ }^{4}$, Yutaka Morita ${ }^{5}$, Yasuyuki Nakamura ${ }^{1,6}$, Katsuyuki Miura ${ }^{1}$ \\ and Hirotsugu Ueshima ${ }^{1,2}$
}

Brachial-ankle pulse wave velocity (baPWV) is a relatively new non-invasive measure of arterial stiffness obtained using an automated system. We assessed the relationship between baPWV and 6.5-year overall mortality from the Takashima cohort study. The baPWV was measured in $\mathbf{2 6 4 2}$ participants at baseline. When participants were divided into tertiles, all-cause mortality increased significantly as baPWV increased $(P<0.001)$. Participants in the highest baPWV tertile showed an increased risk of all-cause mortality evidenced by a multivariable adjusted hazard ratio of 6.8 (95\% confidence interval: 1.4-32.8) as compared with the lowest tertile. The present study demonstrated that increased baPWV is an independent predictor of all-cause mortality in the Japanese population.

Hypertension Research (2010) 33, 922-925; doi:10.1038/hr.2010.103; published online 17 June 2010

Keywords: brachial-ankle pulse wave velocity; longitudinal cohort study; predictor of mortality

\section{INTRODUCTION}

Among the non-invasive and simple methods used to quantify the elastic properties of arteries, pulse wave velocity (PWV) is the most recognized and established index of arterial stiffness. ${ }^{1,2}$ There is a variety of methods used to ascertain the PWV; the most frequently used methods to date have been carotid-femoral PWV (cfPWV) and brachial-ankle PWV (baPWV). ${ }^{3,4}$ Tanaka et al. ${ }^{4}$ recently compared cfPWV with baPWV and found a strong positive association between the two, indicating that both measures of PWV are indices of central arterial stiffness. They also reported that both indices had a similar association with established cardiovascular risk factors. The cfPWV has been reported to have predictive value for future mortality. 5,6 Although there are a few studies reporting the prognostic value of baPWV among patient populations, ${ }^{7,8}$ the predictive value of baPWV for future mortality in the general population has not been evaluated thus far. However, given the simplicity of the technique, baPWV could be ideal for large-scale population screening and could be incorporated into routine clinical settings if its probable predictive value could be identified. To assess the relationship between baPWV and all-cause mortality, we conducted a prospective analysis using the Takashima study, which is a general population-based longitudinal cohort study.

\section{METHODS}

The Takashima study

The present analysis was conducted as a part of the Takashima study, which is a population-based prospective cohort study initiated in 2002 with the intention of investigating lifestyle traits, genetic profile and other risk factors related to cardiovascular disease and to cancer. The cohort participants were recruited from the governmental health check-up in Takashima County located in central Japan. All the participants gave their written informed consent to participate in the study. Baseline information on the history of cardiovascular disease, diabetes, use of anti-hypertensive and anti-diabetic medication, and smoking and drinking habits was obtained from interviews by well-trained public health nurses. Serological measures including lipid profile, blood glucose level and hemoglobin Alc were determined by use of standard techniques. All deaths occurring in the study population during the follow-up period of 6.5 years were identified using data from the National Vital Statistics.

\begin{abstract}
Measurement of baPWV
Together with sphygmomanometric blood pressure measurements, baPWV was measured using a validated automatic device (BP-203RPE II form PWV/ABI; OMRON Healthcare, Tokyo, Japan) in 2642 participants. This instrument simultaneously records the baPWV and the brachial and ankle blood pressures on the left and right sides, produces an electrocardiogram and records the heart sounds. Each participant was examined in the supine position after resting for
\end{abstract}

\footnotetext{
${ }^{1}$ Department of Health Science, Shiga University of Medical Science, Shiga, Japan; ${ }^{2}$ Lifestyle-Related Disease Prevention Center, Shiga University of Medical Science, Shiga, Japan; ${ }^{3}$ Center for Biomedical Ethics \& Law, University of Tokyo, Tokyo, Japan; ${ }^{4}$ Department of Internal Medicine, Takashima General Hospital, Shiga, Japan; ${ }^{5}$ Makino Hospital, Takashima, Shiga, Japan and 6 Department of Cardiovascular Epidemiology, Kyoto Women's University, Kyoto, Japan Correspondence: Dr TC Turin, Department of Health Science, Shiga University of Medical Science, Seta Tsukinowa-cho, Otsu City, Shiga 520-2192, Japan. E-mail: turin@belle.shiga-med.ac.jp

Received 15 January 2010; revised 14 April 2010; accepted 16 April 2010; published online 17 June 2010
} 
at least $5 \mathrm{~min}$. Electrocardiogram electrodes were placed on both wrists, and cuffs were placed on the brachium and the ankles bilaterally. A microphone for detecting heart sounds was placed on the left edge of the sternum. The cuffs were connected to both a plethysmographic sensor that determined the volume pulse form and an oscillometric pressure sensor that measured blood pressure. The brachial and ankle pulse-volume waveforms were recorded using a semiconductor pressure sensor. The baPWV values on the right and left sides were obtained, and the left and right values were averaged for analysis in this study. Details of the device function and process have been described elsewhere. ${ }^{9,10}$

\section{Statistical analysis}

After excluding participants with a history of cardiovascular disease, there were 2480 participants $(839$ men, mean age $61.0 \pm 12.4$ years; and 1641 women, mean age $57.1 \pm 12.8$ years). Participants with baPWV were divided into tertiles and analyzed, with the lowest tertile serving as the reference category. Tertiles were defined as follows: baPWV $<14 \mathrm{~m} \mathrm{~s}^{-1}, 14 \mathrm{~m} \mathrm{~s}^{-1} \leqslant$ baPWV $<17 \mathrm{~m} \mathrm{~s}^{-1}$ and baPWV $\geqslant 17 \mathrm{~m} \mathrm{~s}^{-1}$. For the categorical variables, we used the $\chi^{2}$ statistic to examine differences between the baPWV tertile groups. One-way analysis of variance was used to detect differences in continuous variables across the baPWV tertiles. The Kaplan-Meier product limit method was used to estimate the unadjusted survival curves for the three baPWV groups. The log-rank test was used to compare the equality of survival distributions for the different levels of baPWV. Cox proportional hazards analysis was used to assess the association between baPWV tertiles and mortality risk. Hazard ratios and $95 \%$ confidence intervals are reported. Age, gender, body mass index, high-density lipoprotein-cholesterol, low-density lipoprotein-cholesterol, systolic blood pressure, use of anti-hypertensive and anti-diabetic drugs, serum triglyceride level, hemoglobin Alc, and history of smoking and drinking habits were adjusted for in the multivariate analysis. A value of $P<0.05$ was considered significant. The Omnibus tests of model coefficients were used to test the model fit for the models. These tests demonstrated that the data fit the model adequately. All analyses were performed with the SPSS 17.0 statistical software package (SPSS, Chicago, IL, USA).

\section{RESULTS}

The baseline characteristics of the participants in each baPWV tertile are shown in Table 1a. Age, body mass index, blood pressure indices, low-density lipoprotein-cholesterol, triglyceride and hemoglobin Alc increased, while high-density lipoprotein-cholesterol decreased, as the baPWV value increased. Within the follow-up period of 13788.7 person-years, 59 deaths occurred. The mortality rate per 10000 person-years is shown inTable $1 \mathrm{~b}$. The mortality for the highest tertile was significantly higher than that for the lowest tertile. Figure 1 shows the cumulative probabilities of death by baPWV tertile according to the Kaplan-Meier product limit method. In the Kaplan-Meier analysis, the cumulative hazard risk for all-cause mortality was progressively and significantly greater with increasing baPWV tertile $(P<0.001$ by log-rank test). Table $1 \mathrm{c}$ shows the age and gender adjustment, and the multivariate-adjusted hazard ratios for higher levels of baPWV with reference to the lowest tertile. The highest baPWV category showed an

Table 1 Baseline characteristics and mortality risk according to baPWV tertiles: the Takashima study, Japan

\begin{tabular}{|c|c|c|c|c|}
\hline & Tertile 1 & Tertile 2 & Tertile 3 & P-value \\
\hline \multicolumn{5}{|l|}{ (a) } \\
\hline Range of baPWV & $<14 \mathrm{~ms}^{-1}$ & 14 to $<17 \mathrm{~m} \mathrm{~s}^{-1}$ & $\geqslant 17 \mathrm{~ms}^{-1}$ & \\
\hline Participants & 841 & 824 & 815 & \\
\hline Age (years) & $47.3 \pm 11.4$ & $60.1 \pm 9.2$ & $68.1 \pm 7.4$ & $<0.001$ \\
\hline Men (\%) & 24.7 & 34.7 & 42.3 & $<0.001$ \\
\hline BMI $\left(\mathrm{kg} \mathrm{m}^{-2}\right)$ & $22.5 \pm 2.9$ & $23.1 \pm 2.9$ & $23.3 \pm 2.9$ & $<0.001$ \\
\hline $\mathrm{SBP}(\mathrm{mm} \mathrm{Hg})$ & $114.6 \pm 14.1$ & $129.0 \pm 16$ & $143.2 \pm 20.9$ & $<0.001$ \\
\hline $\mathrm{DBP}(\mathrm{mm} \mathrm{Hg})$ & $69.7 \pm 9.6$ & $78.1 \pm 10.4$ & $83.6 \pm 12.3$ & $<0.001$ \\
\hline Anti-hypertensive medication (\%) & 2.3 & 13.3 & 31.20 & $<0.001$ \\
\hline HDL-cholesterol (mg per 100 ml) & $62.8 \pm 15.7$ & $61 \pm 15.8$ & $58.3 \pm 15.0$ & $<0.001$ \\
\hline LDL-cholesterol (mg per 100 ml) & $121.0 \pm 31.4$ & $130.4 \pm 33.6$ & $126.8 \pm 32.8$ & $<0.001$ \\
\hline Total cholesterol (mg per $100 \mathrm{ml}$ ) & $201.3 \pm 34.2$ & $212.7 \pm 34.9$ & $206.7 \pm 34.9$ & $<0.001$ \\
\hline Triglyceride (mg per $100 \mathrm{ml}$ ) & $85.9 \pm 53.0$ & $105.2 \pm 65.8$ & $106.7 \pm 54.5$ & $<0.001$ \\
\hline $\mathrm{HbAlc}(\%)$ & $4.8 \pm 0.54$ & $5 \pm 0.56$ & $5.2 \pm 0.84$ & $<0.001$ \\
\hline Anti-diabetic drug (\%) & 0.1 & 1.7 & 3.3 & $<0.001$ \\
\hline Current smoker (\%) & 18.3 & 15.7 & 17.5 & 0.01 \\
\hline Current drinker (\%) & 42.3 & 40.4 & 38.5 & NS \\
\hline \multicolumn{5}{|l|}{ (b) } \\
\hline Person-years & 4716.5 & 4645.6 & 4426.7 & \\
\hline Number of all-cause deaths & 2 & 9 & 48 & \\
\hline Mortality rate & $4.2(0.7-14.0)$ & $19.4(9.4-35.5)$ & $108.4(80.9-142.6)$ & \\
\hline \multicolumn{5}{|l|}{ (c) } \\
\hline \multirow[t]{2}{*}{ Age- and sex-adjusted HR } & 1 & $2.3(0.5-11.0)$ & $8.1(1.8-36.9)$ & \\
\hline & & $P=0.301$ & $P=0.007$ & \\
\hline \multirow[t]{2}{*}{ Multivariate-adjusted HR } & 1 & $2.1(0.4-10.2)$ & $6.9(1.4-32.8)$ & \\
\hline & & $P=0.359$ & $P=0.016$ & \\
\hline
\end{tabular}

Abbreviations: BMI, body mass index; DBP, diastolic blood pressure; HbAlc, hemoglobin Alc; HDL-cholesterol, high-density lipoprotein cholesterol; HR, hazard ratio; LDL-cholesterol, low-density lipoprotein cholesterol; SBP, systolic blood pressure.

Values are shown as means \pm s.d., proportions are expressed as percentage. $P$-values are from analysis of variance or chi-square test. Mortality rate is shown per 10000 person-years and $95 \%$ confidence intervals in parentheses. Multivariate HR adjusted for age, sex, BMI, HDL-cholesterol, LDL-cholesterol, SBP level, history of anti-hypertensive and anti-diabetic medication, serum triglyceride level, hemoglobin Alc, history of smoking and drinking habits using Cox proportional hazards model. 


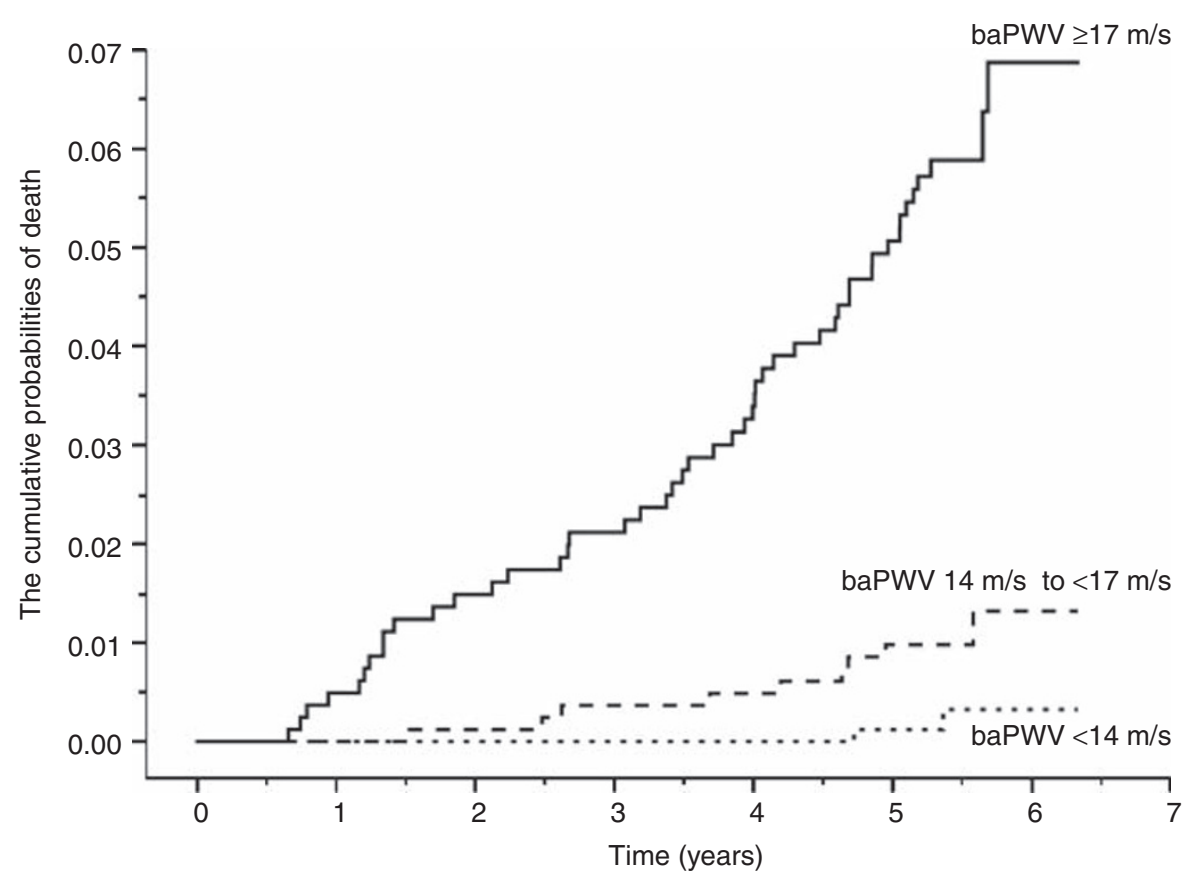

Figure 1 The cumulative probabilities of death by baPWV tertile according to the Kaplan-Meier product limit method were progressively greater as the tertiles increased. The dotted line represents the baPWV $<14 \mathrm{~m} \mathrm{~s}^{-1}$ category (lowest tertile), the broken line represents the $14 \mathrm{~m} \mathrm{~s}^{-1}<$ baPWV $<17 \mathrm{~ms}^{-1}$ category (middle tertile), and the continuous line represents the bAPWV $\geqslant 17 \mathrm{~m} \mathrm{~s}^{-1}$ category (highest tertile). The hazard functions showed a significant difference for test of equality of survival distributions for the different levels of baPWV $(P<0.001)$ by log-rank test.

increased risk of all-cause mortality, as evidenced by the multivariateadjusted hazard ratio of 6.8 (95\% confidence interval: $1.4-32.8$ ) compared with the lowest tertile. In multivariate models, this association of baPWV with all-cause mortality was independent of age, sex, body mass index, high-density lipoprotein-cholesterol, low-density lipoprotein-cholesterol, systolic blood pressure, history of anti-hypertensive or anti-diabetic drug use, serum triglyceride, hemoglobin Alc, and history of smoking and drinking habits.

\section{DISCUSSION}

Although aortic PWV is accurate and reproducible, it may not be ideal for routine use in clinics or for population-based risk screening. The use of pressure transducers or Doppler probes on target arteries may be perceived as somewhat difficult by clinical staff. In addition, people may feel uncomfortable exposing the inguinal area during the acquisition of femoral pressure waveforms. baPWV is a simpler method, requiring simple placement of blood pressure cuffs on the four extremities. Comparing baPWV with cfPWV as a tool to be used in general practice or for population-based screening, baPWV measurements do not require a specialized technique, and the examiner must only place the cuffs on the brachium and ankle. ${ }^{9,10}$ It has been shown that the measured baPWV is strongly associated with the measured cfPWV. ${ }^{4}$ Previous reports suggest that baPWV may provide qualitatively similar information to aortic PWV. ${ }^{11}$ The validity and reproducibility of baPWV measurements have been reported to be as good as those of aortic PWV or cfPWV measurements. ${ }^{9}$

We observed that higher baPWV values were associated with an increased risk for overall death, and this association was independent of subject-level covariates. As the number of patients in the current study was relatively small and the follow-up duration was relatively short, the results of the present study should be confirmed using a larger number of participants and a long-term follow-up period. It would also be valuable to explore the relationship between the measured baPWV and long-term cardiovascular survival. Given the simplicity of the technique and its probable predictive value for future mortality independent from blood pressure, baPWV would be ideal for large-scale population screening and for incorporation into routine clinical settings.

\section{CONFLICT OF INTEREST}

The authors declare no conflict of interest. The Takashima study is partially funded by OMRON Healthcare.

1 Laurent S, Cockcroft J, Van Bortel L, Boutouyrie P, Giannattasio C, Hayoz D, Pannier B, Vlachopoulos C, Wilkinson I, Struijker-Boudier H. Expert consensus document on arterial stiffness: methodological issues and clinical applications. Eur Heart J 2006; 27: 2588-2605.

2 O'Rourke MF, Staessen JA, Vlachopoulos C, Duprez D, Plante GE. Clinical applications of arterial stiffness; definitions and reference values. Am J Hypertens 2002; 15: 426-444.

3 Mancia G, De Backer G, Dominiczak A, Cifkova R, Fagard R, Germano G, Grassi G, Heagerty AM, Kjeldsen SE, Laurent S, Narkiewicz K, Ruilope L, Rynkiewicz A, Schmieder RE, Struijker-Boudier H, Zanchetti A. 2007 Guidelines for the management of arterial hypertension: the task force for the management of arterial hypertension of the European Society of Hypertension (ESH) and of the European Society of Cardiology (ESC). J Hypertens 2007; 25: 1105-1187.

4 Tanaka H, Munakata M, Kawano Y, Ohishi M, Shoji T, Sugawara J, Tomiyama H, Yamashina A, Yasuda H, Sawayama T, Ozawa T. Comparison between carotid-femoral and brachial-ankle pulse wave velocity as measures of arterial stiffness. $J$ Hypertens 2009; 27: 2022-2027.

5 Willum-Hansen T, Staessen JA, Torp-Pedersen C, Rasmussen S, Thijs L, Ibsen H, Jeppesen J. Prognostic value of aortic pulse wave velocity as index of arterial stiffness in the general population. Circulation 2006; 113: 664-670.

6 Inoue N, Maeda R, Kawakami H, Shokawa T, Yamamoto H, Ito C, Sasaki H. Aortic pulse wave velocity predicts cardiovascular mortality in middle-aged and elderly Japanese men. Circ J 2009; 73: 549-553.

7 Tomiyama H, koji Y, Yambe M, Shiina K, Motobe K, Yamada J, Shido N, Nobuhiro T, Taishiro C, Yamashina A. Brachial-ankle pulse wave velocity is a simple and independent predictor of prognosis in patients with acute coronary syndrome. Circ J 2005; 69: 815-822. 
8 Kitahara T, Ono K, Tsuchida A, Kawai H, Shinohara M, Ishii Y, Koyanagi H, Noguchi T, Matsumoto T, Sekihara T. Impact of brachial-ankle pulse wave velocity and anklebrachial blood pressure index on mortality in hemodialysis patients. Am J Kidney Dis 2005; 46: 688-696.

9 Yamashina A, Tomiyama H, Takeda K, Tsuda H, Arai T, Hirose K, Koji Y, Hori S, Yamamoto Y. Validity, reproducibility, and clinical significance of noninvasive brachial-ankle pulse wave velocity measurement. Hypertens Res 2002; 25: 359-364.
10 Tomiyama H, Yamashina A, Arai T, Hirose K, Koji Y, Chikamori T, Hori S, Yamamoto Y, Doba N, Hinohara S. Influences of age and gender on results of noninvasive brachialankle pulse wave velocity measurement-a survey of 12517 subjects. Atherosclerosis 166; 2: 303-309.

11 Sugawara J, Hayashi K, Yokoi T, Cortez-Cooper MY, DeVan AE, Anton MA, Tanaka H. Brachial-ankle pulse wave velocity: an index of central arterial stiffness? J Hum Hypertens 2005; 19: 401-406. 